\title{
Neonatal Kidney Dimensions and Medullary Pyramid Thicknesses According to the Weight, Length and Body Mass Index of Newborns
}

\author{
(1) Hatice Ayça Ata Korkmaz1, @ Şebnem Kader2 \\ 1University of Health Sciences, Trabzon Kanuni Training and Research Hospital, Clinic of Radiology, Trabzon, Turkey \\ ${ }^{2}$ Karadeniz Technical University Faculty of Medicine, Department of Neonatology, Trabzon, Turkey
}

\begin{abstract}
Aim: The primary purpose of the present study was to investigate the relationship between neonatal kidney dimensions and medullary pyramid thicknesses (MPTs) according to the weight, length and body mass index (BMI) of newborns.

Materials and Methods: A total of 237 [128 (54\%) boys and 109 (46\%) girls] consecutive term newborns in the first month of life were evaluated. Infants with an underlying renal disease, urinary symptoms, chromosomal abnormalities or low birth weight were excluded from the study. Greyscale ultrasound of neonatal kidneys was performed in standard transverse and longitudinal planes. Renal dimensions, parenchymal thickness and MPT were also evaluated and compared with neonatal age, height, weight, BMI and abdominal circumference (AC) and head circumferences (HC). Regression analyses were performed and graphs were plotted to determine the independent factors for renal parameters.

Results: Statistically significant associations were noted in the renal dimensional measurements among neonatal weight (NW), neonatal length, BMI, $\mathrm{HC}$ and $\mathrm{AC}$ in the infants $(\mathrm{p}<0.0001)$. However, no statistically significant difference was observed between right kidney MPT and NW, HC, AC and BMI. In contrast, a statistically significant association was detected between left kidney MPT and NW, HC, AC and BMI.

Conclusion: Neonatal kidney length, neonatal kidney width and renal parenchymal thickness measurements could be a useful method as an indicator of neonatal growth.

Keywords: Newborn, renal dimensions, parenchymal thickness, medullary pyramid thickness, neonatal weight
\end{abstract}

\section{Introduction}

The development of the kidney is associated with an increase in the number of nephrons. The kidneys of infants with low birth weight are smaller due to the small number of glomeruli and nephrons (1).

The number of nephrons and glomeruli was determined before birth using a biological variation (1). The development of nephrons has been observed to be completed at 32-34 weeks of gestation, and post-partum nephron development does not occur in babies $(2,3)$. The number of nephrons varies from person to person, although it is believed that there are about a million nephrons per kidney in humans (4). Since the kidney parenchyma contains glomeruli and kidney filtration systems, the measurement of renal parenchymal thickness is believed to be a more sensitive indicator of kidney growth (5). Some diseases such as renal cystic diseases, vesicoureteral reflux and ureteropelvic junction obstruction and also genetic background affect renal parenchymal thickness. In previous studies, intrauterine parenchymal growth was evaluated by 
measuring renal parenchymal thickness (6). In addition, renal parenchymal thickness in low birth weight infants versus normal birth weight infants has been investigated (1).

A correct evaluation of kidney size is very important for clinical diagnosis and treatment. In several diseases, changes occur in the size and morphology of the kidneys. It is important to follow up the kidney dimensions of patients ultrasonographically (USG) in cases such as recurrent urinary tract infections, vesicoureteral reflux and neurogenic bladder. Acute kidney damage (AKD) is a common disorder and often has a severe outcome in neonatal intensive care units. The incidence of AKD in newborns is related to the gestational week, birth weight, postnatal weight and the facilities of the neonatal intensive care unit which affects the prognosis (7). Such diseases can affect renal morphology and size by causing parenchymal atrophy or hypertrophy. Kidney length is the quantitative measurement that is most often used for comparing results determined in accordance with the follow-up study of renal size standards (8-10). Therefore, renal evaluation of healthy newborns is very important to understand pathological changes.

Hence, US imaging has been regarded as the gold standard for the evaluation of renal morphology in newborns. A number of studies have been conducted to determine the normal range of kidney size using sonography. To the best of our knowledge, there has been no study with a large sample size investigating the morphology of kidneys, including "renal medullary pyramid length, kidney size and parenchymal thickness" in newborns. Measurements of the kidneys of children and adults have been established using US previously (8,11-14); however, there are limited available data on neonatal renal dimensions and the length of medullary pyramids.

The primary purpose of the present study was to investigate the relationship between neonatal kidney dimensions and medullary pyramid thicknesses (MPTs) according to the weight, length (NL) and body mass index (BMI) of newborns in a large series.

\section{Materials and Methods}

\section{Study Population}

The study patients were sampled from the patient population treated at the Department of Neonatology in the University of Health Sciences, Training and Research Hospital, Trabzon between January 2016 and September 2016. A total of 249 individuals were seen in our Radiology department, and renal US evaluation was performed for those full-term babies (i.e. having completed 37 weeks of gestation) without urinary tract symptoms or underlying renal problems. Infants with congenital chromosomal abnormalities, those born by caesarean section and those small for gestational age were excluded. A total of 12 infants were excluded from the study because of abnormalities incidentally detected by sonographic evaluation (7 hydronephrosis, 1 nephrocalcinosis and 4 surrenal haematoma). Finally, a total of 237 infants [128 (54.0\%) boys and 109 (46.0\%) girls] were evaluated.

The study was approved by the local Institutional Review Board, and all participants' parents provided written informed consent. This prospective, single-institution study was conducted in compliance with the Declaration of Helsinki and the good clinical practice guidelines of the Ministry of Health of Turkey. The study was approved by the Local Ethics Committee of Kanuni Training and Research Hospital, Trabzon, Turkey (approval number: 2016/10 date 09/03/2016).

All ultrasound examinations were performed within the first month after birth using an Aplio 500 ultrasound machine (Toshiba Medical Systems, Co., Ltd., Otawara, Japan) equipped with linear $4.8-$ to $11-\mathrm{MHz}$ transducers. All the examinations were performed by a radiologist (H.A.A.K.) with more than 10 years of experience in neonatal imaging. The examinations were performed according to international recommendations and guidelines for the safe use of diagnostic ultrasound in medicine (15).

\section{Ultrasound Imaging}

After breastfeeding from the mother, the babies were made to relax and prepared for the US examination. A clean examination bed was prepared before each examination for the protection of babies against contamination. Probes containing baby skin solutions with no harmful effects were used for disinfection. For the comfort of the newborns, bottle warmers were used to maintain a constant body temperature to make the ultrasound procedures more comfortable.

We initially started with B-mode sonographic imaging using an Aplio 500 ultrasound machine (Toshiba Medical Systems, Co. Ltd, Otawara, Japan) equipped with linear 4- to 9-MHz transducers. The aim of using linear transducers was to detect more detail in the anatomic images. After selecting the linear transducers, we selected the neonatal abdomen mode and then selected wide screen trapezoid mode for visualizing the upper and inferior pole of the kidney at the same time with a best resolution. The depth was zoomed in to adjust for a perfect image of the medullary pyramids. If necessary, we increased the focus degree but not the number of foci that were placed at the level of kidney. Finally, we adjusted for a perfect view of the neonatal kidney.

The patient was placed in the contralateral decubitus position over a special pillow that was built for the comfort of the babies. Greyscale US evaluation of both kidneys was performed in standard transverse and longitudinal planes. The maximum vertical and transverse dimensions of each kidney were measured by the examiner (Figure 1).

Renal medullary pyramid length was also measured in the longitudinal plane. Echogenicity and incidental pathologies were evaluated. 


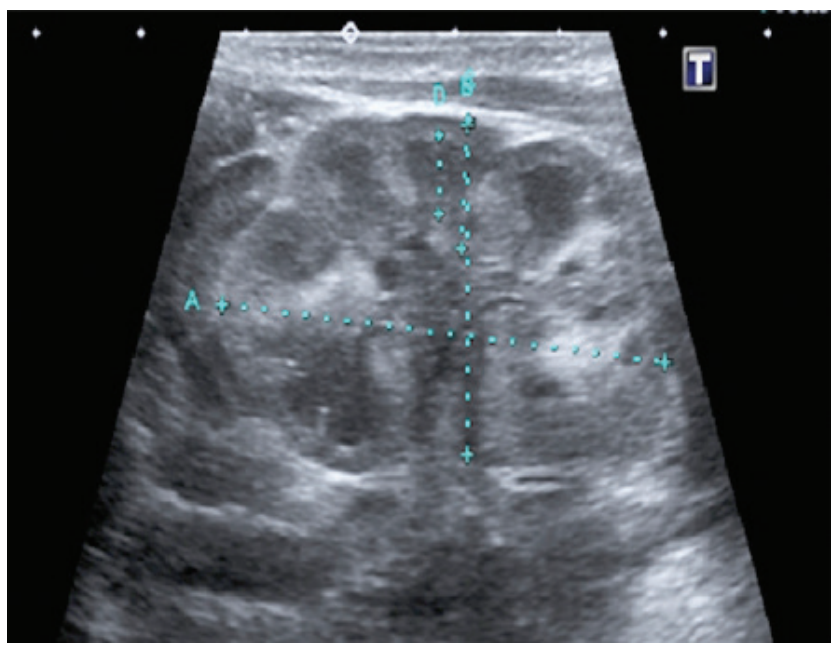

Figure 1. Gray scale evaluation method for neonatal kidney. The maximum vertical A) and transverse B) dimensions, parenchymal thickness C) and medullary pyramidal thickness (D) of kidney was measured

\section{Statistical Analysis}

Statistical analysis was conducted using SPSS 22.0 statistical software.

Continuous variables were expressed as mean \pm standard deviation, and categorical variables were expressed in percentages. Regression analyses were performed to determine the independent factors for renal parameters. Multiple linear regression analysis was performed to analyze the relationship between USC measurements and somatic measurements. Pearson's correlation test was used to evaluate the correlations of continuous variables, and $p$ values $<0.05$ were considered as statistically significant.

\section{Results}

A total of 237 term newborns comprising 109 (46\%) girls and 128 (54\%) boys were evaluated in this study. Mean gestational age, weight and height of the term infants were $39.2 \pm 1.01$ weeks, $3.534 \pm 519 \mathrm{~g}$ and $50.6 \pm 1.08 \mathrm{~cm}$, respectively. The clinical and demographic characteristics of the patients are shown in Table I.

Mean head circumference $(\mathrm{HC})$, abdominal circumference (AC) and BMI were $35.6 \pm 0.90 \mathrm{~cm}, 38.0 \pm 2.26 \mathrm{~cm}$ and $0.22 \pm 0.02$, respectively.

A significant association was observed between right kidney length (RKL) and neonatal weight (NW) ( $r=0.259$, $p<0.0001)$. Furthermore, the relationship between right kidney width (RKW)-NW $(r=0.382, p<0.0001)$ and right kidney parenchymal thickness-NW $(r=0.241, p<0.0001)$ was also statistically significant.

Other neonatal clinical variables such as NL, HC, BMI and AC also showed a statistically significant association between both kidney diameters apart from the right kidney MPT.

\begin{tabular}{|l|l|}
\hline \multicolumn{2}{|l|}{ Table I. The clinical and demographic characteristics of patients } \\
\hline Variables & Mean \pm SD \\
\hline Age (week) & $39.2 \pm 1.01$ \\
\hline NW $(\mathrm{g})$ & $3534 \pm 519$ \\
\hline NL $(\mathrm{cm})$ & $50.6 \pm 1.08$ \\
\hline HC $(\mathrm{cm})$ & $35.6 \pm 0.90$ \\
\hline BMI $(\mathrm{cm})$ & $0.22 \pm 0.02$ \\
\hline AC $(\mathrm{cm})$ & $38.0 \pm 2.26$ \\
\hline RKL $(\mathrm{cm})$ & $45.01 \pm 4.83$ \\
\hline RKW $(\mathrm{cm})$ & $21.20 \pm 3.20$ \\
\hline RKPT $(\mathrm{cm})$ & $9.1 \pm 1.10$ \\
\hline RKMPT $(\mathrm{cm})$ & $5.60 \pm 3.20$ \\
\hline LKL $(\mathrm{cm})$ & $45.17 \pm 5.82$ \\
\hline LKW $(\mathrm{cm})$ & $21.34 \pm 2.86$ \\
\hline LKPT $(\mathrm{cm})$ & $9.27 \pm 1.06$ \\
\hline LKMPT $(\mathrm{cm})$ & $5.86 \pm 1.07$ \\
\hline SD S & \\
\hline
\end{tabular}

SD: Standard deviation, NW: Neonatal weight, NL: Neonatal length, HC: Head circumference, BMI: Body mass index, AC: Abdominal circumference, RKL: Right kidney length, RKW: Right kidney width, RKPT: Right kidney parenchymal thickness, RKMPT: Right kidney medullary pyramidal thickness, LKL: Left kidney length, LKW: Left kidney width, LKPT: Left kidney parenchymal thickness, LKMPT: Left kidney medullary pyramidal thickness

The regression analysis graphs depicting the relationships between left kidney length (LKL)-NL and RKW-NW are shown in Figure 2,3.

The correlation results between renal parameters and clinical variables are shown in Table II. Regarding the independent factors for renal parameters, the regression analyses revealed that NW (95\% Cl: 0.000-0.004, $\mathrm{p}=0.029)$ and $\mathrm{HC}(95 \% \mathrm{Cl}: 0.120-1.704, \mathrm{p}=0.024)$ were independent markers of RKL. In addition, NL (95\% Cl: 0.780-2.546, $\mathrm{p}<0.0001$ ) was found to be an independent marker of $L K L$.

\section{Discussion}

The present study has shown that renal dimensions, parenchymal thickness and left kidney MPT are related to the BMI and weight of the infant. The size of the kidneys and the medullary parenchymal thickness showed no association with age in the first 30 days of life.

The renal parenchyma consists of the renal cortex and the medullary pyramids. These structures may also be evaluated using computed tomography or magnetic resonance imaging (MRI), as well as by ultrasound (12,13). MRI, which is an expensive imaging modality, is particularly difficult to administer in children because of the requirement of sedation. Conversely, US is a non-invasive, cost-effective and easily accessible imaging modality that can be used for the rapid evaluation of neonatal kidneys. US also does not involve ionizing radiation (11). 
In newborns, physical examination methods such as palpation and percussion are inadequate to evaluate kidney

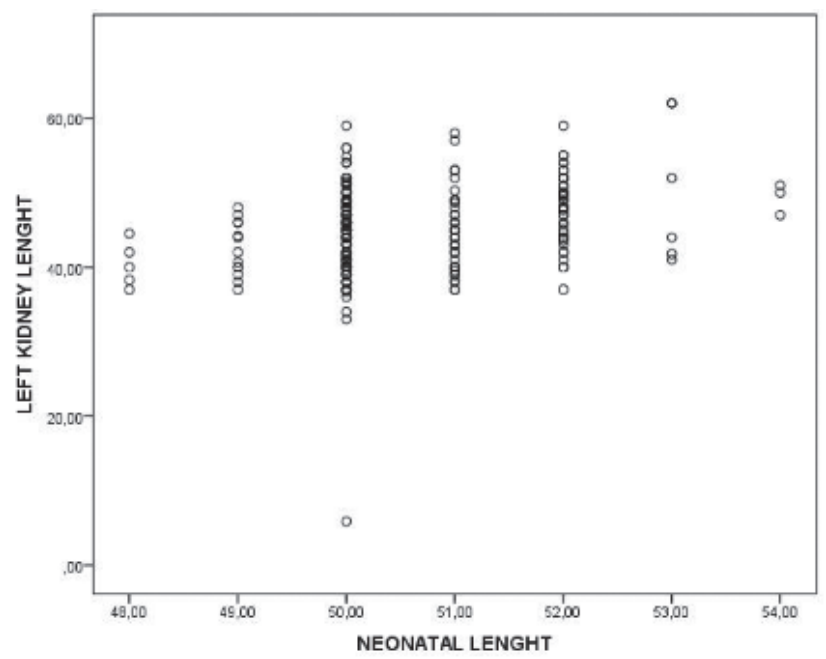

Figure 2. Regression graph for left kidney length and neon natal length

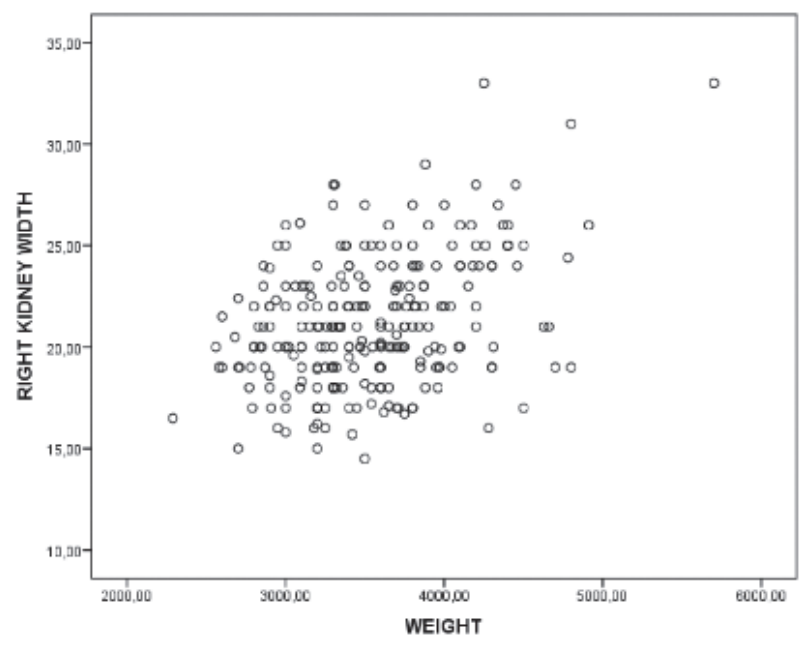

Figure 3. Regression graph for right kidney width and neonatal weight size. Radiography, intravenous pyelography and radionuclide studies are not preferred in routine practice for the evaluation of neonatal kidneys due to radiation risks and difficulties in application. In addition, renal diameters assessed by conventional radiological investigations other than US may be about $16 \%$ larger than their actual size. Several factors may be responsible for this oversizing, such as the centration of the tube and the change in distance from the patient (photographic magnetization) or because of respiration, the osmotic effect of the contrast agent (chemical magnetization) or various other reasons such as renal axis variations.

A previous study reported that weight indicated the best correlation with neonatal kidney measurements in the first week of life (16). However, only 1-week newborns were included and renal parenchymal thickness and MPT were not evaluated in that study. Furthermore, although $\mathrm{HC}, \mathrm{AC}$ and $\mathrm{BMI}$ are important measurements for the evaluation of infant development in newborn intensive care units, that study did not include these parameters.

Some studies have reported that renal parenchymal thickness might be used as a measure of renal growth in low birth weight infants versus normal birth weight infants (1). However, the smallest functional unit of the kidney is the renal glomerulus and the renal glomerulus present in the cortex. The renal parenchymal thickness is the sum of the thicknesses of the cortex and the medullary pyramids. Therefore, MPT must also be assessed to calculate the functional unit, i.e. the cortex.

There is little information regarding the relationship between MPT and BMI. Eze et al. (17) found a weak correlation between MPT and BMI and a moderate positive correlation between MPT and weight. However, we did not find any association between MPT and BMI in our study. There may be several explanations for this discrepancy. First, the study populations are different. Eze et al. (17) included patients aged up to 18 years, and neonatal populations accounted for a very small proportion of that population. In contrast, our study

Table II. Correlation between renal parameters and clinical variables

\begin{tabular}{|l|l|l|l|l|l|}
\hline Variables & NW (g) & NL (cm) & HC (cm) & BMI (cm) & AC (cm) \\
\hline RKL $(\mathrm{cm})$ & $r=0.259$ & $r=0.226$ & $r=0.305$ & $r=0.260$ & $r=0.273$ \\
\hline RKW $(\mathrm{cm})$ & $r=0.382$ & $r=0.325$ & $r=0.247$ & $r=0.251$ & $r=0.342$ \\
\hline RKPT $(\mathrm{cm})$ & $r=0.241$ & $r=0.152$ & $r=0.125$ & $r=0.194$ & $r=0.141$ \\
\hline RKMPT (cm) & $r=0.037$ & $r=0.019$ & $r=0.029$ & $r=0.020$ & $r=0.030$ \\
\hline LKL (cm) & $r=0.244$ & $r=0.340$ & $r=0.203$ & $r=0.155$ & $r=0.224$ \\
\hline LKW (cm) & $r=0.381$ & $r=0.274$ & $r=0.216$ & $r=0.325$ & $r=0.368$ \\
\hline LKPT $(\mathrm{cm})$ & $r=0.313$ & $r=0.189$ & $r=0.209$ & $r=0.319$ & $r=0.266$ \\
\hline LKMPT (cm) & $r=0.238$ & $r=0.161$ & $r=0.163$ & $r=0.252$ & $r=0.198$ \\
\hline
\end{tabular}

NW: Neonatal weight, NL: Neonatal length, HC: Head circumference, BMI: Body mass index, AC: Abdominal circumference, RKL: Right kidney length, RKW: Right kidney width, RKPT: Right kidney parenchymal thickness, RKMPT: Right kidney medullary pyramidal thickness, LKL: Left kidney length, LKW: Left kidney width, LKPT: Left kidney parenchymal thickness, LKMPT: Left kidney medullary pyramidal thickness 
population is exclusively neonatal and the population size is relatively larger than their study population. Second, genetic factors might have a potential impact on the evolution of MPT.

\section{Conclusion}

In conclusion, we have demonstrated that renal dimensional measurements and parenchymal thickness can also be used as a marker for evaluating postnatal growth of a newborn.

Renal dimensional measurements and parenchymal thickness could be a useful method as an indicator of neonatal growth. In newborns, kidney measurements are independent from postnatal age but related with the development of the baby. Therefore, renal nomograms need to be determined according to the somatic parameters of neonates.

\section{Declaration of Interest}

The authors declare that there is no conflict of interest that could be perceived as prejudicing the impartiality of the research reported.

\section{Ethics}

Ethics Committee Approval: The study was approved by the University of Health Sciences Kanuni Training and Research Hospital Local Ethics Committee (approval number: 2016/10 date 09/03/2016).

Informed Consent: All participants' parents provided written informed consent.

Peer-review: Externally peer-reviewed.

\section{Authorship Contributions}

Concept: H.A.A.K., Design: H.A.A.K., Ş.K., Data Collection or Processing: H.A.A.K., Ş.K., Analysis or Interpretation: H.A.A.K., Writing: H.A.A.K.

Conflict of Interest: No conflict of interest was declared by the authors.

Financial Disclosure: The authors declared that this study received no financial support.

\section{References}

1. Brennan S, Kandasamy Y. Renal parenchymal thickness as a measure of renal growth in low-birth-weight infants versus normal-birth-weight infants. Ultrasound Med Biol 2013;39:231520.
2. Bagby SP. Developmental origins of renal disease: should nephron protection begin at birth? Clin I Am Soc Nephrol 2009;4:10-3.

3. Askenazi D), Ambalavanan N, Goldstein SL. Acute kidney injury in critically ill newborns: what do we know? What do we need to learn? Pediatr Nephrol 2009;24:265-74.

4. Hoy WE, Douglas-Denton RN, Hughson MD, Cass A, Johnson $\mathrm{K}$, Bertram JF. A stereological study of glomerular number and volume: preliminary findings in a multiracial study of kidneys at autopsy. Kidney Int Suppl 2003:31-7.

5. Hricak H, Slovis T, Callen C, Callen P, Romanski R. Neonatal kidneys: sonographic anatomic correlation. Radiology 1983;147:699-702.

6. Hadar E, Davidovits M, Mashiach R, et al. Sonographic evaluation of kidney parenchymal growth in the fetus. Arch Gynecol Obstet 2012;286:867-72.

7. Timovska SN, Cekovska S, Tosheska-Trajkovska K. Acute kidney injury in newborns. Pril (Makedon Akad Nauk Umet Odd Med Nauki) 2015;36:83-9.

8. Rosenbaum D, Korngold E, Teele RL. Sonographic assessment of renal length in normal children. AJR Am / Roentgenol 1984;142:467-9.

9. Klare B, Geiselhardt B, Wesch H, Schärer K, Immich H, Willich E. Radiological kidney size in childhood. Pediatr Radiol 1980;9:15360.

10. Han BK, Babcock DS. Sonographic measurements and appearance of normal kidneys in children. AJR Am J Roentgenol 1985;145:611-6.

11. Christophe C, Cantraine F, Bogaert C, et al. Ultrasound: a method for kidney size monitoring in children. Eur J Pediatr 1986;145:532-8

12. Dinkel $E$, Ertel $M$, Dittrich $M$, Peters $H$, Berres $M$, SchulteWissermann $\mathrm{H}$. Kidney size in childhood sonographical growth charts for kidney length and volume. Pediatr Radiol 1985;15:3843.

13. Konuş OL, Ozdemir A, Akkaya A, Erbaş G, Celik H, Işik S. Normal liver, spleen, and kidney dimensions in neonates, infants, and children: evaluation with sonography. AJR Am / Roentgenol 1998;171:1693-8.

14. Zerin JM, Blane CE. Sonographic assessment of renal length in children: a reappraisal. Pediatr Radiol 1994;24:101-6.

15. Barnett SB, Ter Haar GR, Ziskin MC, Rott H-D, Duck FA, Maeda $\mathrm{K}$. International recommendations and guidelines for the safe use of diagnostic ultrasound in medicine. Ultrasound Med Biol 2000;26:355-66

16. Soyupak SK, Narli N, Yapicioğlu H, Satar M, Aksungur EH. Sonographic measurements of the liver, spleen and kidney dimensions in the healthy term and preterm newborns. Eur ) Radiol 2002;43:73-8.

17. Eze CU, Akpan VP, Nwadike UI. Sonographic assessment of normal renal parenchymal and medullary pyramid thicknesses among children in Enugu, Southeast, Nigeria . Radiography 2016;22:25-31 